\title{
Volume Bragg Grating Formation in Fused Silica with High Repetition Rate Femtosecond Yb:KGW Laser Pulses
}

\author{
Domas PAIPULAS, Viačeslav KUDRIAŠOV, Kęstutis KURŠELIS, Mangirdas MALINAUSKAS, Simone OST, \\ Valdas SIRUTKAITIS
}

\author{
Vilniaus University, Laser Research Center, Saulètekio av. 10, LT-10223 Vilnius, Lithuania \\ E-mail: domas.paipulas@ff.stud.vu.lt
}

\begin{abstract}
In this work we present results on volume Bragg grating formation inside the pure fused silica with the $\mathrm{Yb}: \mathrm{KGW}$ femtosecond laser, operating at $300 \mathrm{kHz}$ repetition rate and having pulse duration of 300 fs. Gratings were created by direct laser writing technique, translating the sample perpendicular to the focused laser beam and forming several grating layers in order to achieve diffraction efficiencies up to $57 \%$. The influence of writing pulse energy and the sample scanning speed on structure morphology are also discused. We have demonstrated that high repetition rate helps to decrease the manufacturing time of such devices: $1 \mathrm{~mm} \times 1 \mathrm{~mm}$ grating, having $2 \mu \mathrm{m}$ period, with thicknesses up to $60 \mu \mathrm{m}$ was written in less than an hour.
\end{abstract}

DOI:10.2961/jlmn.2010.03.0007

Keywords: femtosecond micromachining, volume Bragg gratings, refractive index change, fused silica.

\section{Introduction}

By tightly focusing femtosecond laser radiation inside the bulk of fused silica, it is possible to induce highly localized structural changes with modified optical properties. Depending on laser pulse energy, different type of structures can be created: at low intensities, the zones of smoothly modified refractive index (type I) are created [1]; at medium intensity values, the modifications become anisotropic and possess strong birefringence (type II), it was demonstrated that this property appears due to nanograting formation inside the pulse affected zone [2,3]; and with high pulse intensities microexplosions and void-like structures can appear in the bulk of the processed material [4]. The key processes of this light-material interaction are nonlinear multiphoton absorption followed by avalanche ionization that takes place in the focused region. Absorbed light rapidly heats and, afterwards, remelts the material to different state having modified refractive index [5]. This is considered as the main source for the modification appearance, however, other physical phenomena, like color center formation [6] and structural changes caused by material densification [7], also influence the magnitude and type of refractive index change. Nevertheless, the result is the deposition of a significant fraction of the laser pulse energy within the small focal volume inside the dielectric material. By moving the focal position inside the transparent material it is possible to fabricate various three-dimensional photonic structures such as waveguides [1], diffractive components [8], data storage elements [4] and even, with the aid of chemical treatment, complicated microchannels [9].

\section{Volume Bragg gratings}

The ability to locally change the refractive index of fused silica opens wide opportunities to various photonic applications, previously possible only to limited choice of photosensitive materials. One of such photonic devices is the Volume Bragg Grating (VBG). These gratings have many advantages compared with common amplitude or profile gratings.

Traditionally, VBGs with high diffraction efficiencies are fabricated using a holographic technique. The pattern generated by two beam interference process is recorded in a photosensitive material. The most popular material used today for such devices is DiChromated Gelatin (DCG), which has a very high refractive index modulation property ( $\Delta n$ up to 0.1 ), while retaining good transparency in a broad spectral range. With this technology it is possible to form large area gratings with spatial frequencies up to $6000 \mathrm{~mm}^{-1}$. After the exposure, the gelatin is chemically treated and sandwiched between glass plates for better robustness and handling [10]. A new and very promising material for VBG fabrication is PTR (Photo-ThermoRefractive) glass. It is a silicate based glass with silver, cerium and fluorine doping. After UV illumination and thermal treatment, it can exhibit a refractive index change of up to $10^{-3}$. That change is reasonably smaller than for DCG, but it is more stable and frequencies up to $10000 \mathrm{~mm}^{-1}$ can be reached [11].

Fused silica is one of the most popular materials used in optical systems; it is a widely available and reasonably inexpensive glass. The holographic method is not suited for VBG fabrication in pure fused silica glass as high laser intensities are required to reach the refractive index modification regime; however, the point by point Direct Laser Writing (DLW) technique can be successfully applied for devices fabrication. This technique has advantages over the holographic method as any desired three-dimensional patterns can be fabricated. The manufacturing speed, however, is significantly slower than the holographic method. 
Several attempts to manufacture VBGs in pure fused silica by means of femtosecond laser radiation were reported during last decade $[13,14]$. The efficiencies of these devices were low, hardly reaching $30 \%$. The main problem that researchers encountered was an unpredictable refractive index modification level in fused silica without a clearly identified cause. This parameter is crucial for the devise efficiency. To our knowledge, the VBGs made in pure fused silica and having highest efficiencies (74.8\%) were manufactured by Yamada at al. [14]. These gratings were fabricated by modifying thick regions of the sample using a filamentation process. However, this technique does not have flexibility to control the modified zone size thus preventing the optimization of device fabrication. Another drawback is slow fabrication speed - to form a complete, sub-millimeter size grating requires roughly six hours.

In this work we demonstrate the possibility to manufacture VBGs with relatively high efficiencies in less than an hour by employing high repetition rate femtosecond $\mathrm{Yb}: \mathrm{KGW}$ laser pulses.

\subsection{The diffraction efficiency of VBG}

VBGs with high diffraction efficiencies have become an important tool in optical systems, especially in those where spectral and angular selectivity is essential. In contrast with amplitude or profile gratings, diffraction in phase gratings is produced from periodic modulation of the refractive index created in the bulk of a transparent material. The VBGs theory is described using a coupled wave model devised by Kogelnik [15].

The transmitting grating formed in the bulk of the sample is shown in Fig. 1. Black stripes here represent region of sinusoidally modulated refractive index. The modulation magnitude is $\Delta n$, the period of the grating - $d$ and the thickness - $t$. An incident beam $I_{\text {inc }}$, enters the grating at angle $\alpha_{i n c}$. The diffraction condition for this grating is the same as in ordinary surface grating:

$$
\frac{m \lambda}{n_{a v} d}=\sin \alpha_{i n c}-\sin \alpha_{d i f},
$$

here $m$ is an integer, corresponding to the order of diffraction, $\lambda$ is wavelength, $n_{a v}$ is the refractive index of the material and $\alpha_{d i f}$ is the angle of diffracted beam. In contrast with ordinary grating, VBG have additional dimension of thick-

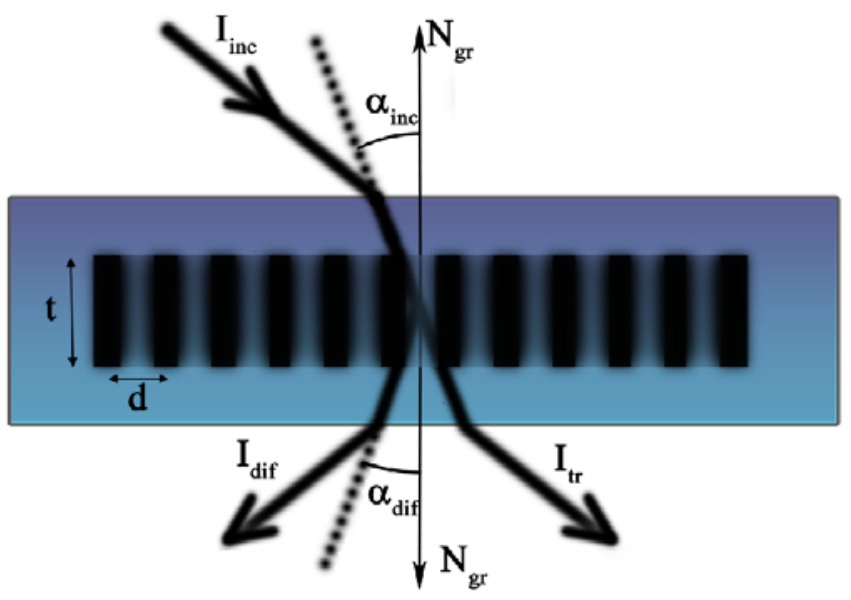

Fig. 1 Schematics of transparent volume phase grating. The equally spaced black stripes represent the region of sinusoidally modulated refractive index. ness with which it is possible to control the efficiency of diffracted beam $\left(I_{d i}\right)$. The VBG diffraction efficiency, described as ratio between diffracted and incident beams, is given by:

$$
\eta=\sin ^{2}\left(\frac{\pi \Delta n t}{\lambda \cos \alpha_{i n c}}\right) .
$$

This equation is only valid for angles that satisfy the Bragg condition, $\alpha_{i n c}=\alpha_{\text {dif }}$.

The VBG period $(d)$ and the wavelength $(\lambda)$ alone determine the angle at which Bragg condition is satisfied, while the refractive index modulation level $(\Delta n)$ and thickness $(t)$ determine the efficiency of the structure. For ideal conditions it is theoretically possible to achieve $100 \%$ diffraction efficiency by choosing optimal design parameters. As efficiency is a pure sinusoidal function periodic in the product $\Delta n \bullet t$, for a given $\Delta n$ value, only gratings with specific thickness could operate with high diffraction efficiency. That thickness ranges from several microns for high refractive index modulation level $(\Delta n>0.1)$ up to several hundred microns for low level $(\Delta n<0.001)$. If the modulation level of the refractive index is unknown, the thickness of the VBG has to be found experimentally.

\section{Experimental setup and results}

\subsection{Experimental setup}

We used a regeneratively amplified $\mathrm{Yb}: \mathrm{KGW}$ laser ("Pharos", made by "Light Conversion Ltd.") as femtosecond radiation source. This laser produces high intensity pulses with $1030-\mathrm{nm}$ wavelength, at $300-\mathrm{fs}$ duration and variable repetition frequency up to $300 \mathrm{kHz}$. The conventional optical setup used in the experiment is shown in Fig. 2. Laser pulses are first attenuated and then focused into the bulk of fused silica with a $0.42-N A$ lens, having focal length of $6.24 \mathrm{~mm}$. The sample was mounted on a 3-axis "Aerotech" based nanopositioning system ("AltSCA" assembled by "Altechna Ltd.") having positioning accuracy up to $50 \mathrm{~nm}$. The structures where formed by translating sample perpendicular to the laser beam with varying speeds from 0.1 to $20 \mathrm{~mm} / \mathrm{s}$. The modifications in fused silica (KU1) and fused quartz (KV) where induced with laser pulse energies in the range from $0.1 \mu \mathrm{J}$ to $2.00 \mu \mathrm{J}$. In order to minimize the influence of spherical aberrations all structures were written no deeper than $400 \mu \mathrm{m}$ bellow the sample surface.

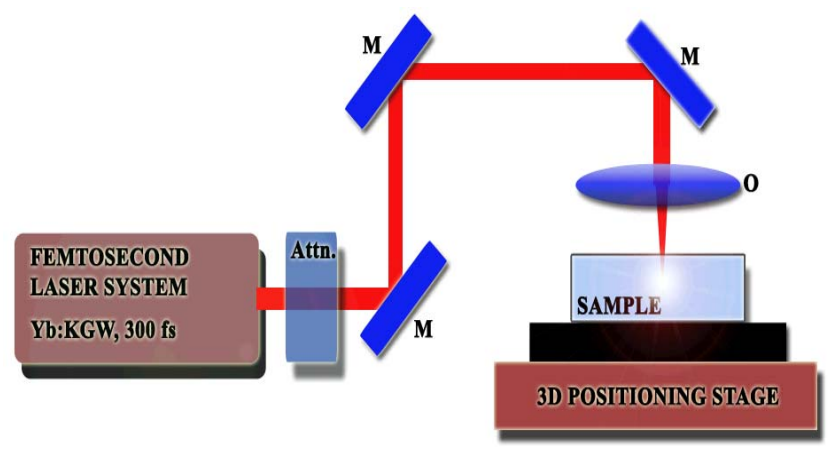

Fig. 2 Optical setup for grating fabrication. M denotes guiding mirrors, Attn. - attenuation system consisting of polarizer and phase plate, $\mathrm{O}-$ objective. Laser beam diameter - $3.6 \mathrm{~mm}$. 
3.1 The influence of pulse energy and scanning speed on the structure morphology

Pulse writing energies and sample translating speed play major roles in uniformity and quality of modified regions. The optimal laser pulse energies and scanning speeds have to be estimated experimentally. For this task, single line structures where fabricated and analyzed under a transmitting microscope. Visible modifications in fused silica were observed with average pulse energies higher than $0.15 \mu \mathrm{J}$ (threshold fluency $-2.6 \mathrm{~J} / \mathrm{cm}^{2}$ ). By increasing the intensity, the modifications become more prominent; however, the uniformity of these structures tends to decrease (Fig. 3). The smallest, smooth structures were achieved with pulse energies in range of 0.2-0.3 $\mu \mathrm{J}$. Structure diameters of $1.7 \mu \mathrm{m}$ were slightly lower than the theoretical beam waist at the focus $(2.3 \mu \mathrm{m})$, showing the nonlinear origin of absorption mechanism. Higher intensities lead to thicker structure creation, but regularity tends to decreases due to the appearance of different modification types. The polarization also influences the structure morphology: smooth and even structures were created only with polarization parallel to translation direction. Similar results were obtained with a fused quartz sample; the only difference was slightly lower (approx. by $20 \%$ ) fluency threshold value for modification, which can be explained by higher impurity content of the quartz glass.

The big drawback of DLW technique used in transparent material processing is the slow fabrication speed. Using a conventional $1-\mathrm{kHz}$ laser system, the sample must be translated slower than $1 \mathrm{~mm} / \mathrm{s}$ to allow modified regions to overlap. Single-pulse-induced modifications, however, do not possess good characteristics, and multipulse treatment is required for the uniform remelting of the affected zone. Multipulse processing also determines the magnitude of the refractive index modulation level. So in practice, the optimal scanning speed is lower and reaches only tenths and even hundredths of millimeters per second.

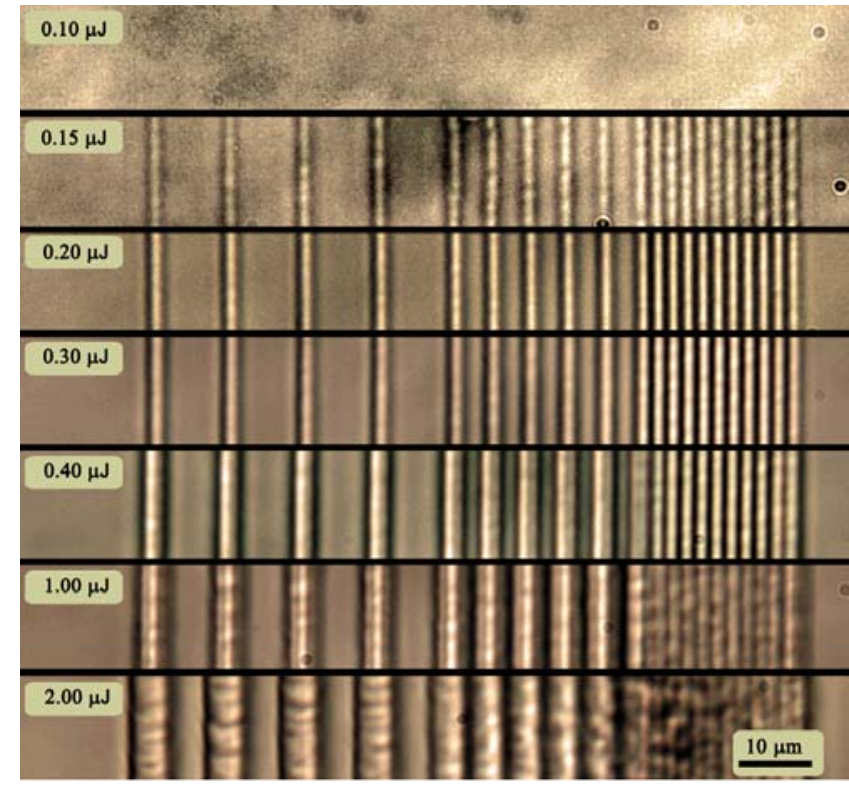

Fig. 3 Modifications induced in fused silica at various pulse energies. Sample scanning speed $0.1 \mathrm{~mm} / \mathrm{s}$, focusing lens $0.42 \mathrm{NA}$, repetition rate $100 \mathrm{kHz}$, polarization parallel to scanning direction. Line groups in the left are separated by $10 \mu \mathrm{m}$, in the middle by $5 \mu \mathrm{m}$, in the right by $2 \mu \mathrm{m}$. Samples were written $200 \mu \mathrm{m}$ below surface.



Fig. 4 Modifications induced in fused silica at different scanning speeds. Writing pulse energy $0.2 \mu \mathrm{J}$, repetition rate $100 \mathrm{kHz}$, focused with $0.42 \mathrm{NA}$ lens. Samples were written $200 \mu \mathrm{m}$ below surface.

The influence of scanning speed on the modification morphology is demonstrated in Fig. 4. It is clear that, even with high-repetition-rate irradiation (in this case $100 \mathrm{kHz}$ ),

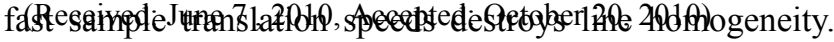
Smooth structures can be fabricated only when scanning speed is less than $1 \mathrm{~mm} / \mathrm{s}$. This clearly demonstrates the accumulation effect: knowing that single-pulse modified zone is $\sim 1.7 \mu \mathrm{m}$ in diameter, the minimum number of pulses required to affect the processed zone should be no less than 150 in order to get continuously modified lines. This ratio does not depend on the pulse repetition rate at least up to $300 \mathrm{kHz}$. At $5 \mathrm{kHz}$ the maximum scanning speed was found to be $50 \mu \mathrm{m} / \mathrm{s}$, while at $300 \mathrm{kHz}$ it was $3 \mathrm{~mm} / \mathrm{s}$. This suggest that this accumulation effect is not linked with cumulative heating at the focal position as the time scale is too long and during pulse-to-pulse arrival time, the zone being processed is able to dissipate all acquired heat.

\subsection{VBG fabrication}

The schematic structure of the fabricated grating inside the fused silica is shown in Fig. 5. The period of the grating was chosen to be $2 \mu \mathrm{m}$. In such configuration each line would slightly overlap, achieving a more close-tosinusoidal refractive index modulation pattern. Sufficient thickness of the VBG is created by fabricating several layers of gratings on top of each other with some spacing (in our case the spacing was chosen to be $4.5 \mu \mathrm{m}$ which guarantees slight overlap of the layers overlaps). The lines were 


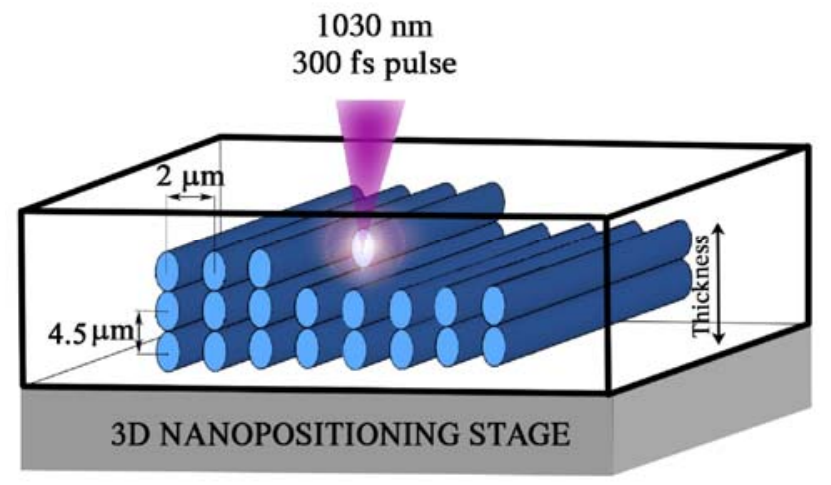

Fig. 5 Schematic layout of fabricated VBG.

recorded with average pulse energies from $0.15 \mu \mathrm{J}$ to $0.25 \mu \mathrm{J}$ and the sample-translating speed was set to $3 \mathrm{~mm} / \mathrm{s}$ at $300-\mathrm{kHz}$ pulse repetition rate. The first grating layer was formed $400 \mu \mathrm{m}$ below the sample's surface.

As the precise magnitude of refractive index modulation was unknown, several $1 \mathrm{~mm} \times 1 \mathrm{~mm}$ gratings with different thicknesses were fabricated. Their diffraction efficiencies are shown in Fig. 6a. The efficiency was measured with 633-nm wavelength at angles satisfying Bragg condition thus giving the highest efficiency value. The measured Bragg angle was $6.2^{\circ}$ and it perfectly coincides with theoretical value. The detuning from this condition results in rapid drop of the efficiency (Fig. 6b). The maximum diffraction efficiency of $57 \%$ was observed in a grating with $70-\mu \mathrm{m}$ thickness produced with $0.20-\mu \mathrm{J}$ laser writing energy. We used a white-light continuum filtered with interference filters to learn how this grating performed at different wavelengths. The results are shown in Fig. 6c. The measured efficiencies at specific wavelengths were slightly lower in this experiment, and efficiency reached around $40 \%$ for the unfiltered broad spectrum. Measurement errors were larger with the filtered light due to the low incident beam intensity compared to HeNe laser output. It is important to stress, that for high efficiency the grating should be positioned at the Bragg angle for each wavelength.

By increasing (or decreasing) grating thickness a drop in efficiency was observed. This suggests that $70-\mu \mathrm{m}$ thickness is an optimal thickness which produces highest diffraction for given refractive index modulation level. The refractive index modulation level obtained from (2) is 0.005 . By increasing writing power up to $0.25 \mu \mathrm{J}$ the overall efficiency slightly drops; however, maximum efficiency is observed at the same thickness. This suggests that by increasing intensity no higher levels of uniform refractive index modification can be achieved, but material damage starts to appear, increasing scattering level. When writing energy is lower, the modulation level is also smaller, so for $0.15 \mu \mathrm{J}$ the optimal thickness has to be higher and was not evaluated in this experiment. The manufacturing time of the VBG depends on the number of grating layers; a single $1 \mathrm{~mm} \times 1 \mathrm{~mm}$ layer is fabricated in less than 3 minutes, though $70-\mu \mathrm{m}$-thickness gratings are fabricated in less than 45 minutes.

There are two main reasons that prevent higher diffraction efficiencies of fabricated VBGs. First is scattering of the incident beam from the fabricated structure caused by

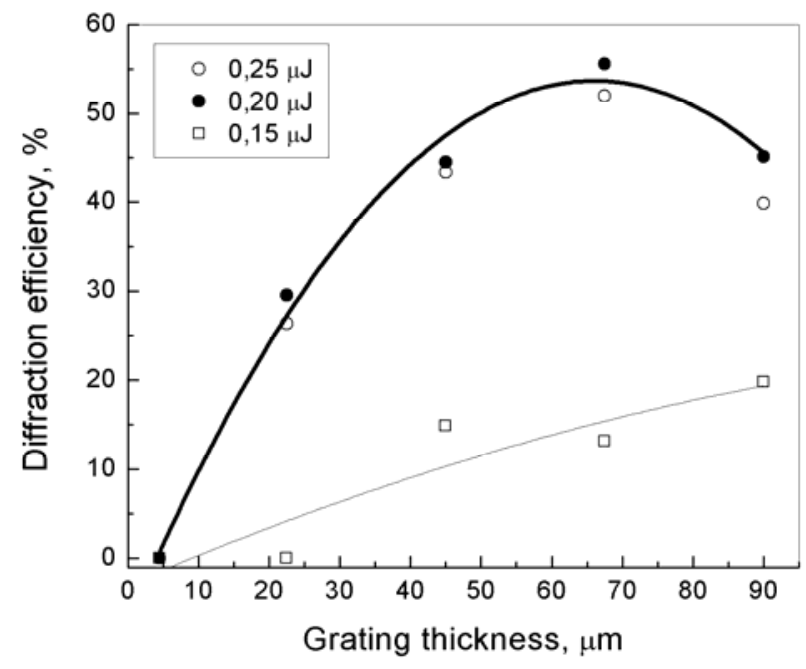

a)

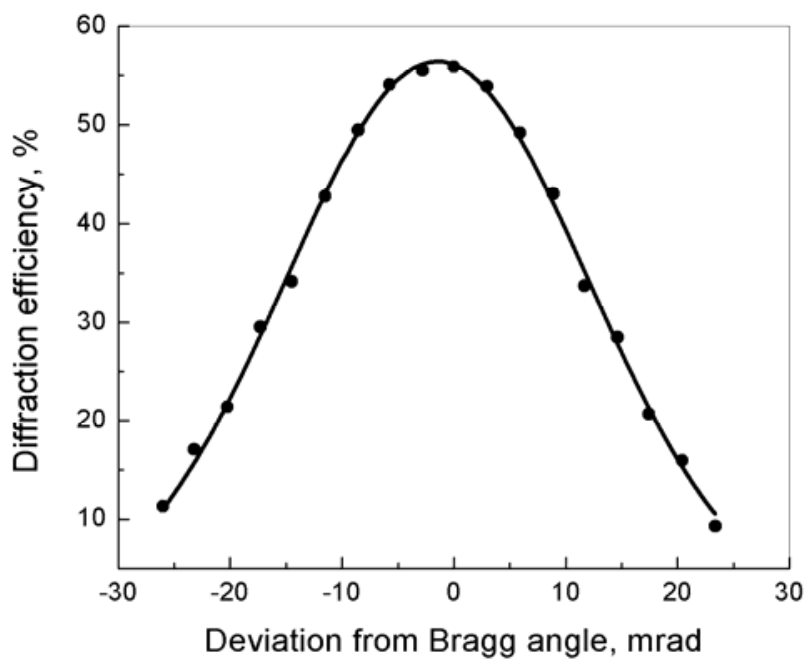

b)

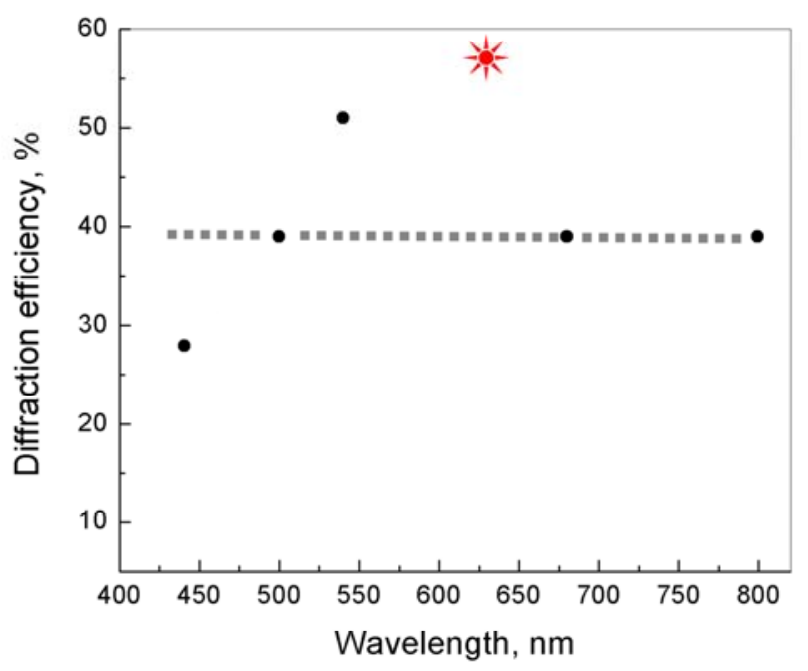

c)

Fig. 6 Diffraction efficiency dependence: a) on the grating thickness written with different pulse energies; b) on the detuning from the Bragg angle; c) on the incident wavelength measured with white light continuum (at Bragg conditions), the star shows the efficiency of 633$\mathrm{nm}$, wavelength. All curves are fitted to the experiment data. 
non-uniformity and scattering centers such as microdamages. The second cause is non-sinusoidally modulation of the refractive index change induced in the material. Theoretical models of VBG describe the sinusoidally modulated refractive index; however, it is hard to achieve such distribution using direct laser writing technique as the modification profile after single shot is rather complicated [16]. Optimal writing conditions are best found experimentally, and even higher grating performance could be achieved by parameter optimization.

\section{Conclusions}

We have demonstrated that high pulse repetition rate can help to sufficiently decrease manufacturing time of various photonic devices in fused silica, reaching the sample translation velocities up to millimeters per second. Faster scanning speeds in our experiment tended to destroy structure homogeneity suggesting the importance of accumulation effects that take place during modification process. Faster writing speeds allowed parameter optimization and fabrication of volume Bragg gratings having diffraction efficiencies up to $57 \%$.

\section{Acknowledgments}

This work is supported by the Lithuania State Science and Studies Foundation Grant B09/08 (Laser MicroProcessing with High Repetition Femtosecond Pulses). S.O. is grateful for the Erasmus internship grant. Authors thank dr. R.C. Eckardt for manuscript editing.

\section{References}

[1] K. M. Davis, K. Miura, N. Sugimoto and K. Hirao: Opt. Lett., 21, (1996) 1729.

[2] L. Sudrie, M. Franco, B. Prade and A. Mysyrowicz: Opt. Commun., 191, (2001) 333.

[3] Y. Shimotsuma, P. G. Kazansky, J. Qiu, and K. Hirao: Phys. Rev. Lett., 91, (2003) 247405-1.

[4] E. N. Glezer, M. Milosavljevic, L. Huang, R. J. Finlay, T.-H. Her, J. P. Callan and E. Mazur: Opt. Lett., 21, (1996) 2023.

[5] J. Chan, T. Huser, S. Risbud and D. Krol: Opt. Lett., 26, (2001) 1726.

[6] K. Hirao and K. Miura: J. Non-Cryst. Solids, 239, (1998) 91.

[7] A. M. Streltsov and N. F. Borrelli: J. Opt. Soc. Am. B., 19, (2002) 2496.

[8] T. Toma, Y. Furuya, W. Watanabe, K. Itoh, J. Nishii and K. Hayashi: Opt. Rev., 7, (2000) 14.

[9] Y. Bellouard, A. Said, M. Dugan and P. Bado: Opt. Express, 12, (2004) 2120.

[10] J. A. Arns, W. S. Colburn and S. C. Barden, Proc. of SPIE, 3779, (1999), p. 313

[11] O. M. Efimov, L. B. Glebov and V. I. Smirnov, Opt. Lett., 25, (2000) 1693.

[12] C. Florea and K. Winick: Lightwave Technol., 21, (2003) 246.

[13] T. Tamaki, W. Watanabe, H. Nagai, M. Yoshida, J. Nishii and K. Itoh: Opt. Express, 14, (2006) 6971.

[14] K.Yamada, W. Watanabe, K. Kintaka, J. Nishii and K. Itoh: Jpn. J. Appl. Phys., 42, (2003) 6916.

[15]H. Kogelnik, Bell Syst. Tech. J., 48, (1969) 2909.

[16] A. Mermillod-Blondin, R. Stoian, A. Rosenfeld and I. V. Hertel: JLMN, 1, (2006) 15.

(Received: June 7 , 2010, Accepted: October 20, 2010) 